\section{Adaptive deblocking filter for intrablocks}

\author{
Jungyoup Yang, Kwanghyun Won, and Byeungwoo Jeon \\ Sungkyunkwan University, Department of Electronic and \\ Electrical Engineering, 300 Chunchun-dong, Jangan-gu, \\ Suwon, Republic of Korea \\ E-mail: bjeon@skku.edu
}

\begin{abstract}
The H.264/AVC deblocking filter pays little attention to intracoded blocks. We enhance this filter by extending it to use intraprediction mode information in its adaptive application to the intracoded block. Experiments show its higher coding efficiency, with blocking artifacts sufficiently minimized in intracoded blocks. (C) 2010 Society of Photo-Optical Instrumentation Engineers. [DOI: 10.1117/1.3523479]
\end{abstract}

Subject terms: deblocking filter; intrablock; H.264/AVC; high efficiency video coding.

Paper 100678LR received Aug. 30, 2010; revised manuscript received Nov. 5, 2010; accepted for publication Nov. 10, 2010; published online Dec. 28, 2010.

\section{Introduction}

Block-based video coding techniques have been widely used in many video compression standards such as H.261, H.262, H.263, and H.264/AVC. ${ }^{1}$ Since each picture is divided into small blocks to be predicted, transformed, and quantized, this block-based nature can cause visually annoying effects known as blocking artifacts along block boundaries. The causes are two-fold. One is the quantization taking place individually in blocks. The quantization error in the transform domain affects entire pixels in the corresponding block. ${ }^{2}$ Different quantization over adjacent blocks can produce visually annoying discontinuities on the block boundaries. The other reason is the block-based prediction. Since a block is predicted by its matching block most likely at different locations in a reference picture, its block boundaries may not match well with those of its neighbors. Blocking artifacts seriously decrease the subjective video quality. Furthermore, a blocky reference picture makes the coding efficiency of its subsequent referring picture worse. H.264/AVC employs an adaptive deblocking filter. To adaptively control the strength of the deblocking filter for each block boundary, it selects a boundary strength (BS) ranging from 0 to 4 based on the coding conditions of two adjacent blocks $(P, Q)$, defining the block boundary under processing. ${ }^{1,3} \mathrm{~A}$ larger BS means stronger filtering. When $P$ and $Q$ are intercoded, no filtering $(\mathrm{BS}=0)$ or relatively weak filtering (BS $=1,2$ ) is applied to remove blocking artifacts caused both by prediction (referred by reference picture and motion vector) and quantization (referred by all zero/nonzero transform coded coefficients). When $P$ and/or $Q$ are intracoded, the strongest filtering $(\mathrm{BS}=4)$ is selected when the boundary is an MB boundary, and strong filtering $(\mathrm{BS}=3)$ is selected otherwise. This decision is made irrespective of intracoding conditions such as intraprediction mode and/or its relative direction with respect to block boundary. The work ${ }^{4}$ tried to

0091-3286/2010/\$25.00 (c) 2010 SPIE have more adaptivity at the expense of increased complexity in its directional deblocking filter based on prediction direction.

High efficiency video coding (HEVC), which is currently being developed by the Joint Collaborative Team on Video Coding (JCT-VC), is mainly focusing on coding video having high resolution and high quality. ${ }^{5}$ The high quality suggests that the coded video is more likely to have less blocking artifacts. Furthermore, various intraprediction directions show different patterns of blocking artifacts. It is noted that the H.264/AVC deblocking filter employing large BS values (3, 4) (which is not adaptive to various intracoding conditions) may use a BS stronger than necessary, resulting in much oversmoothing.

This work presents a deblocking filter capable of more adaptive control of BS using the intraprediction modes for intrablocks. Experiments show not only subjective enhancement but also an average bit saving of $2.08 \%$. In particular, it gives more coding gain in high resolution video such as $1920 \times 1080$ sequences.

\section{Proposed Deblocking Filter}

H.264/AVC deblocking filter applies the strongest deblocking filtering ( $\mathrm{BS}=3,4)$ to intracoded blocks, irrespective of their coding conditions. However, such strong deblocking filtering can badly affect not only the coding efficiency of intracoded blocks, but also that of the intercoded blocks that refer to them. To solve this problem, we propose to take into account the intraprediction direction in deblocking filtering of intracoded blocks, as shown in Fig. 1. If $P$ and/or $Q$ are intracoded and their MB types are different, relatively strong filtering with $\mathrm{BS}=3$ is applied, since the boundary between $P$ and $Q$ has strong blocking artifacts. Otherwise, intraprediction modes of $P$ and $Q$ are considered to jointly decide BS. Different intraprediction modes of $P$ and $Q$ indicate that the two blocks are predicted in different directions, thus the effects of prediction and quantization on the blocks are expected to be nonidentical. In this case, normal filtering (BS $=2$ ) is applied. Otherwise, if intraprediction modes of $P$ and $Q$ are the same, the two blocks are most likely to be in the same region. Therefore, in this case, if block boundary and prediction directions are the same, no filtering $(\mathrm{BS}=0)$ is applied. Otherwise, the weakest filtering $(\mathrm{BS}=1)$ is applied. When directions of block boundary and prediction are the same, for example, as shown in Fig. 2(a), the two blocks are most likely to belong to different objects. Thus, no filtering $(\mathrm{BS}=0)$ should be helpful to avoid an unintended smoothing over the block boundary. On the other hand, if intraprediction is performed across the given block boundary, the blocks are most likely to belong to the same object, so some degree of filtering $(\mathrm{BS}=1)$ might help removing the quantization error that otherwise may be visible across the boundary. For this reason, in the proposed method, vertical and horizontal intraprediction modes are considered to check the difference between block boundary and prediction directions. Detailed filtering operations subsequent to the BS decision are the same as H.264/AVC, ${ }^{1,3}$ and their more comprehensive theoretical details can be found in Ref. 3 .

\section{Experimental Results}

The proposed method is implemented on Joint Model (JM) 16.2 reference software and simulated under the call for 


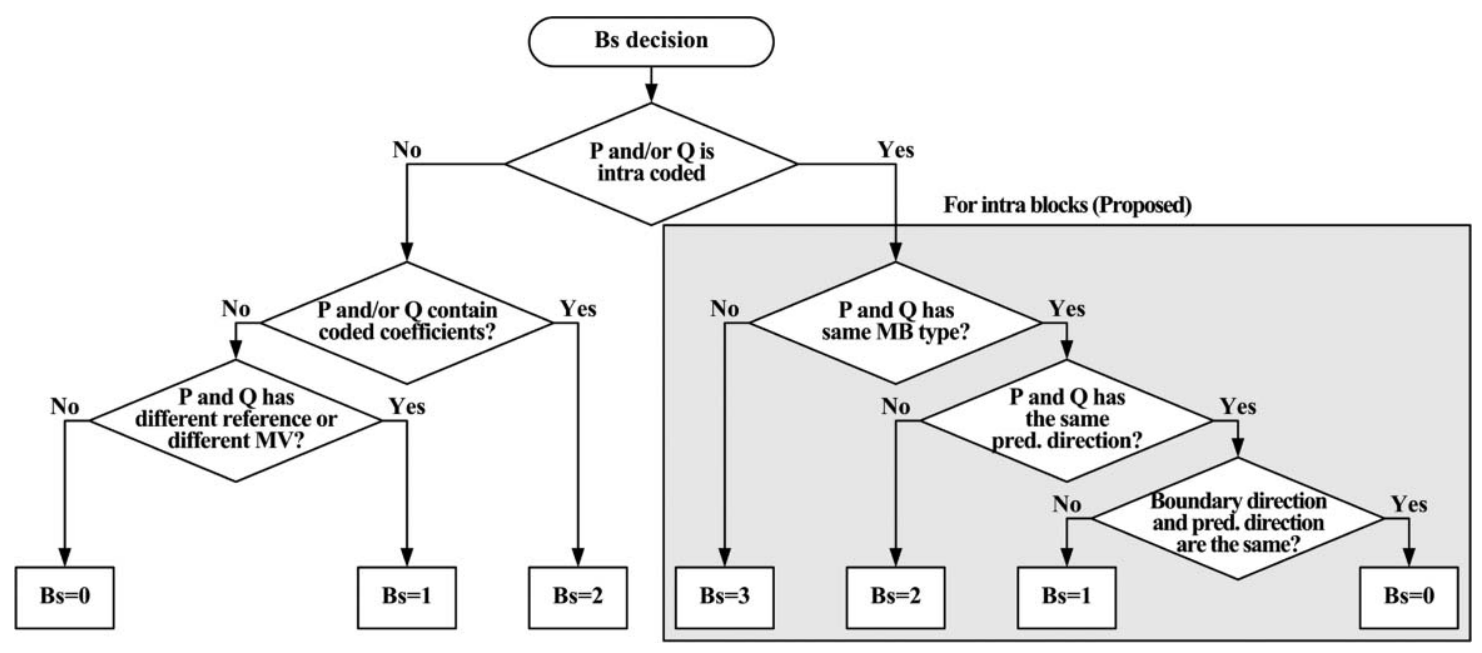

Fig. 1 BS decision of the proposed method.

proposal test condition as determined by JCT-VC in evaluating a new video coding standard for HEVC. ${ }^{6}$ Since the proposed scheme targets the intracoded blocks, it is tested under all intracoding structure. Four quantization parameters (QPs) of 22, 27, 32, and 37 are used, and the coding performance of the proposed scheme is evaluated in both subjective and objective qualities. For the objective quality, the Bjøntegaard delta bit rate $(\mathrm{BDBR})^{7}$ and Bjøntegaard delta peak signalto-noise ratio (BDPSNR) are used against the H.264/AVC deblocking filter in JM 16.2 software as an anchor. These quantities give the average bit rate and PSNR difference of the proposed method compared to the H.264/AVC standard.

Table 1 indicates that the coding gain of the proposed scheme is about $2.08 \%$ on average compared to the anchor. In particular, the proposed scheme gives the best coding gain in $1920 \times 1080$ sequences. Figure 3 shows a subjective quality comparison. Oversmoothing by strong deblocking filtering applied irrespective of intracoded condition by H.264/AVC is remarkably reduced by the proposed method. When directions of block boundary and prediction are the same, the proposed method can keep the vertical edge better by adaptive application of weak- and no-deblocking filters. The benefit is mostly seen when the block boundary coincides with the MB boundary (see Fig. 4).

\section{Conclusions}

We propose a very simple intrablock deblocking filter capable of adaptive assignment of different BS values by

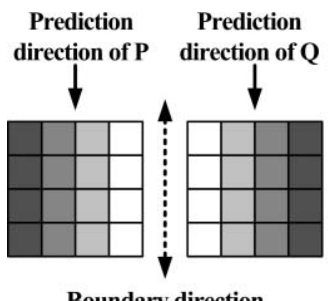

(a)

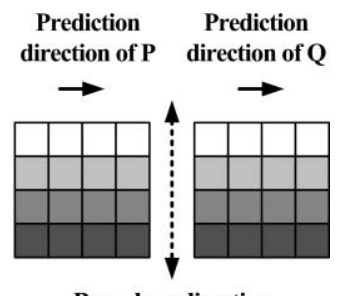

Boundary direction

(b)
Fig. 2 Filtering and prediction directions: (a) same direction and (b) different direction.
Table 1 Objective quality comparison.

\begin{tabular}{|c|c|c|c|}
\hline \multirow[b]{2}{*}{ Size } & \multirow[b]{2}{*}{ Sequence } & \multicolumn{2}{|c|}{ Anchor versus proposed } \\
\hline & & BDPSNR [dB] & BDBR [\%] \\
\hline & ParkScene & 0.162 & -3.262 \\
\hline & Kimono & 0.145 & -3.694 \\
\hline \multirow[t]{5}{*}{$1920 \times 1080$} & Cactus & 0.099 & -2.386 \\
\hline & BasketballDrive & 0.095 & -2.888 \\
\hline & BQTerrace & 0.088 & -1.869 \\
\hline & BasketballDrill & 0.136 & -2.593 \\
\hline & BQMall & 0.101 & -1.682 \\
\hline \multirow[t]{3}{*}{$832 \times 480$} & PartyScene & 0.086 & -1.154 \\
\hline & RaceHorses & 0.121 & -1.947 \\
\hline & BasketballPass & 0.160 & -2.528 \\
\hline \multirow[t]{4}{*}{$416 \times 240$} & BQSquare & 0.094 & -1.139 \\
\hline & BlowingBubbles & 0.090 & -1.243 \\
\hline & RaceHorses & 0.148 & -2.206 \\
\hline & Vidyo1 & 0.102 & -1.863 \\
\hline \multirow[t]{2}{*}{$1280 \times 720$} & Vidyo3 & 0.089 & -1.460 \\
\hline & Vidyo4 & 0.123 & -2.303 \\
\hline Average overall & & 0.114 & -2.080 \\
\hline
\end{tabular}




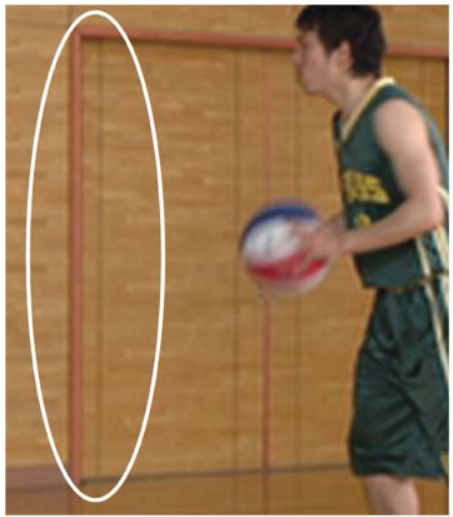

(a)

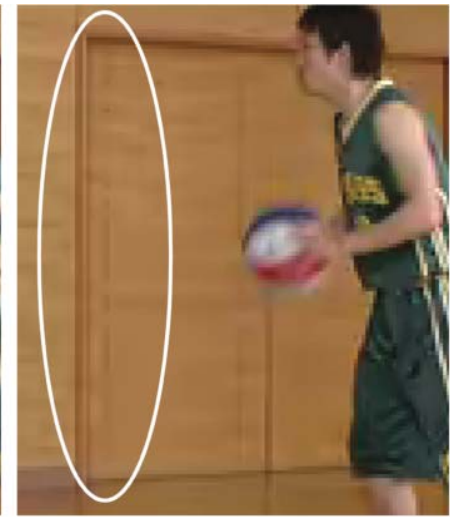

(b)

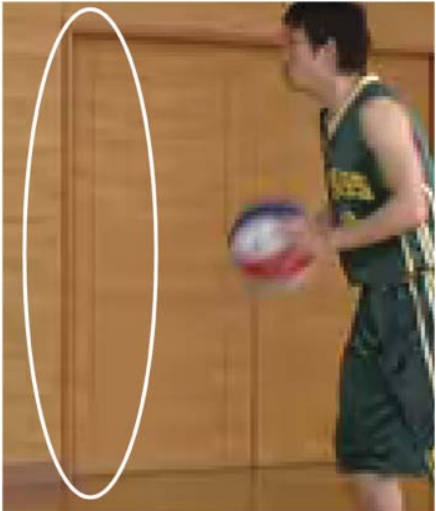

(c)

Fig. 3 Subjective quality of BasketballPass $(416 \times 240)$ with QP32: (a) original, (b) anchor, and (c) proposed.

referring to the intraprediction modes. It achieves an average coding gain of about $2.08 \%$, and increases subjective quality significantly. Since a deblocking scheme more adaptive to various prediction directions other than just vertical and horizontal directions can further improve visual quality, the current simple design may have a drawback. However, our main guideline of designing as simple a deblocking filter as possible leads to the simplest BS decision. A smarter scheme

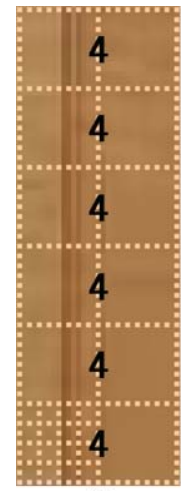

(a)

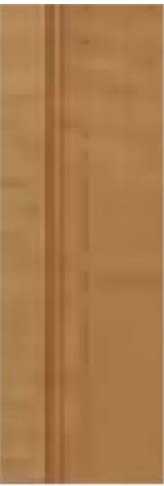

(b)

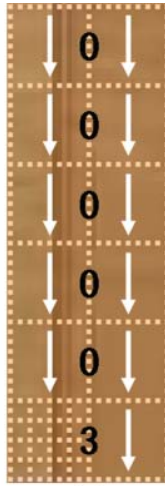

(c)

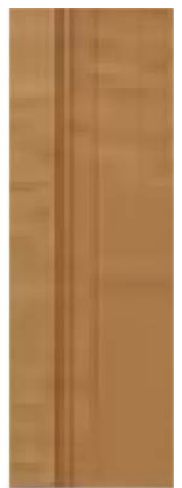

(d)
Fig. 4 Selected regions of Fig. 3: (a) Bs of anchor, (b) anchor, (c) Bs of proposed, and (d) proposed. but with equal or less complexity is one direction of future extension of the proposed scheme.

\section{Acknowledgment}

This research was supported by the Basic Science Research Program through the National Research Foundation of Korea (NRF) funded by the Ministry of Education, Science and Technology (number 20100008030).

\section{References}

1. JVT of ISO/IEC MPEG and ITU-T VCEG, Draft ITU-T Recommendation and Final Draft International Standard of Joint Video Specification, ITU-T Rec. H.264, ISO/IEC 14496-10 AVC, ISO, Geneva, Switzerland (2003).

2. B. Jeon and Jechang Jeong, "Blocking artifacts reduction in image coding based on minimum block discontinuity criterion," IEEE Trans. Circuits Syst. Video Tech. 8(3), 345-357 (Jun 1998)

3. P. List, A. Joch, J. Lainema, G. Bjøntegaard, and M. Karczewicz, "Adaptive deblocking filter," IEEE Trans. Circuit Syst. Video Tech. 13(7), 614-619 (2003).

4. J. Jeong, S. Kim, Y. G. Kim, Y. Choi, and Y. Choe, "A directional deblocking filter based on intra prediction for H.264/AVC," Trans. IEICE Electron. Express 6(12), 864-869 (2009).

5. "Vision, applications and requirements for high-performance video coding," ISO/IEC MPEG Doc. N11096, ISO, Geneva, Switzerland (Jan. 2010).

6. "Joint call for proposals on video compression technology," ISO/IEC MPEG/ITU-T SG16 VCEG Doc. N11113/VCEG-AM91, ISO, Geneva, Switzerland (Jan. 2010).

7. G. Bjøntegaard, "Calculation of average PSNR differences between RD-curves," ITU-T SG16/Q6 Doc. VCEG-M13, ITU, Geneva, Switzerland (Apr. 2001). 